\title{
SIRT1 exerts protective effects against paraquat-induced injury in mouse type II alveolar epithelial cells by deacetylating NRF2 in vitro
}

\author{
YING-WEI DING, GUANG-JU ZHAO, XIAO-LIN LI, GUANG-LIANG HONG, \\ MENG-FANG LI, QIAO-MENG QIU, BIN WU and ZHONG-QIU LU \\ Department of Emergency Medicine, The First Affiliated Hospital \\ of Wenzhou Medical University, Wenzhou, Zhejiang 325000, P.R. China
}

Received October 14, 2015; Accepted February 9, 2016

DOI: $10.3892 /$ ijmm.2016.2503

\begin{abstract}
Silent information regulator 2-related enzyme 1 (SIRT1), a protein deacetylase, is known to strongly protect cells against oxidative stress-induced injury. The nuclear factor E2-related factor 2 (NRF2)-antioxidant response element (ARE) antioxidant pathway plays important regulatory roles in the antioxidant therapy of paraquat (PQ) poisoning. In the present study, we investigated whether the SIRT1/NRF2/ ARE signaling pathway plays an important role in lung injury induced by PQ. For this purpose, mouse type II alveolar epithelial cells (AECs-II) were exposed to various concentrations of PQ. The overexpression or silencing of SIRT1 was induced by transfecting the cells with a SIRT1 overexpression vector or shRNA targeting SIRT1, respectively. The protein expression levels of SIRT1 and NRF2 were measured by western blot analysis. The superoxide dismutase (SOD) and catalase (CAT) activities, as well as the glutathione (GSH) and malondialdehyde (MDA) levels were measured using respective kits. Heme oxygenase-1 (HO-1) activity was also determined by ELISA. In addition, cell apoptosis was determined by flow cytometry. The protein stability of NRF2 was analyzed using cycloheximide and its acetylation in the cells was also determined. The following findings were obtained: i) SIRT1 overexpression markedly increased NRF2 protein expression; ii) SIRT1 promoted the transcriptional activity of NRF2 and upregulated the expression of the NRF2 downstream genes, SOD, CAT, GSH and HO-1, thus inhibiting the apoptosis of AECs-II; iii) the inhibition of SIRT1 activity further induced
\end{abstract}

Correspondence to: Mr. Guang-Ju Zhao or Professor Zhong-Qiu Lu, Department of Emergency Medicine, The First Affiliated Hospital of Wenzhou Medical University, Wenzhou, Zhejiang 325000, P.R. China E-mail: zgj_0523@126.com

E-mail:1zq640815@163.com

Key words: paraquat, silent information regulator 2-related enzyme 1, nuclear factor E2-related factor 2, oxidative stress, deacetylate the production of malondialdehyde (MDA), which resulted in increased oxidative damage; iv) SIRT1 promoted the stability of NRF2 by regulating the deacetylation and activation of the NRF2/ARE antioxidant pathway. The findings of this study demonstrate that the protective effects of SIRT1 are associated with the activation of the NRF2/ARE antioxidant pathway in lung injury induced by PQ poisoning.

\section{Introduction}

Paraquat (PQ) is widely used as a herbicide; however, it is strongly toxic to humans (1). Multiple organ damage is induced by $\mathrm{PQ}$, which is absorbed through the skin, the respiratory tract and the digestive tract (1), and it mainly accumulates in lung cells. The PQ concentration in lung tissue is 6-10-fold higher than that in plasma, and it causes acute pulmonary edema, progressive dyspnea and pulmonary fibrosis (2). PQ poisoning can lead to the production of reactive oxygen species (ROS) and PQ radicals through cyclic reduction/oxidation. Redox cycling is believed to play an essential role in the initiation of lung damage and fibrosis resulting from PQ poisoning (3).

Silent information regulator 2-related enzyme 1 (SIRT1) is a NAD ${ }^{+}$-dependent protein deacetylase (4) and is involved in various cellular processes, including cell proliferation, apoptosis and inflammation (5). Previous studies have confirmed that SIRT1 plays a significant role in lung injury caused by smoking, bronchial asthma and oxidative stress-related lung diseases (6-8).

Nuclear factor E2-related factor 2 (NRF2) is an important transcription factor, which regulates genes associated with the scavenging of oxygen free radicals, and is regarded as an effective target for antioxidant therapy $(9,10)$. Under conditions of oxidative stress, NRF2 translocates to the nucleus, and then induces the gene expression of downstream detoxification enzymes and antioxidant enzymes, such as superoxide dismutase (SOD) and heme oxygenase-1 (HO-1), in order to produce an antioxidant effect (11-13). Previous studies have revealed that SIRT1 promotes the activity of NRF2 and upregulates the expression of NRF2 downstream genes, such as SOD $(14,15)$, and that the knockdown of SIRT1 results in the downregulation of NRF2 expression (16). In our previous 
study, we confirmed that NRF2 is activated and plays a role in protecting A549 lung cells against oxidative damage following exposure to PQ (17). However, the association between SIRT1 and NRF2, as well as their effects on PQ-induced oxidative stress remain to be elucidated.

Therefore, in the present study, we aimed to determine whether the SIRT1/NRF2/ARE signaling pathway plays an important role in lung injury induced by $\mathrm{PQ}$. For this purpose, mouse type II alveolar epithelial cells (AECs-II) were exposed to various concentrations of PQ and the effects of PQ on the levels of antioxidant enzymes were determined. In addition, to examine the association between SIRT1 and NRF2, SIRT1 expression was either induced or silenced by transfection of the cells with a SIRT1 overexpression vector or shRNA targeting SIRT1, respectively.

\section{Materials and methods}

Animals and reagents. ICR mice ( $\mathrm{n}=20$, weighing 18-30 g) of clean grade were provided by the Animal Experimental Center of Wenzhou Medical University (Wenzhou, China). All experimental procedures involving animals were pre-approved in accordance with the Institutional Animal Care and Use Committee guidelines of Wenzhou Medical University. PQ used in this study was purchased from Sigma-Aldrich (St. Louis, MO, USA) and compounded into a liquid of corresponding concentrations. The antibodies, including anti-SIRT1 (\#3931), anti-NRF2 (\#12721), anti-glyceraldehyde-3-phosphate dehydrogenase (GAPDH; \#5174) and anti-acetyl lysine (\#9441) antibodies were obtained from Cell Signaling Technology (Danvers, MA, USA).

Isolation and culture of mouse AECs-II. The mice were sacrificed by cervical dislocation, and both lungs were quickly removed and placed into $75 \%$ ethanol for $5 \mathrm{~min}$. Subsequently, both lungs were moved to a superclean bench and residual tracheal tissue and connective tissue were cleared to the maximum extent possible. Finally, the cleared lung tissues were placed in sterile pre-cold PBS and washed 2 to 3 times. The lung tissues were washed twice in phosphate-buffered saline (PBS) and cut into small sections (approximately $1 \mathrm{~mm}^{3}$ ) and then digested with $0.25 \%$ trypsinase (Gibco, Carlsbad, CA, USA) under gentle agitation at $37^{\circ} \mathrm{C}$. After 5 min, digestion was terminated using RPMI-1640 medium with $10 \%$ fetal calf serum (both from Gibco, Carlsbad, CA, USA). The above process was repeated once. Subsequently, $0.5 \%$ collagenase type I (Sigma-Aldrich) was added at $37^{\circ} \mathrm{C}$ for $15 \mathrm{~min}$, after which the tissues were centrifuged at $1,000 \mathrm{x} \mathrm{g}$ for $5 \mathrm{~min}$ and washed twice with RPMI-1640 medium containing $10 \%$ fetal calf serum. The cells were then transferred to a $25 \mathrm{~cm}^{2}$ culture flask and cultured in a $\mathrm{CO}_{2}$ incubator (Thermo Fisher Scientific, Waltham, MA, USA) at $37^{\circ} \mathrm{C}$.

Transfection and transfection efficiency analysis. For the upregulation or silencing of SIRT1, lentiviral particles to induce the overexpression of SIRT1 and shRNA specific to SIRT1 to silence SIRT1 were synthesized by Shanghai GeneChem Co., Ltd. (Shanghai, China). A lentiviral overexpression vector (LV-SIRT1) and shRNA targeting SIRT1 (shRNA-SIRT1) were used to induce the overexpression of the silencing of SIRT1, respectively. The shRNA-SIRT1 sequence was 5'-GAUGAAGUUGACCUCCUCATT-3'. The cells were seeded in 6-well plates until they grew to approximately $80 \%$ coverage, and solutions of the viral particles equal to a multiplicity of infection (MOI) of 80 were then pre-mixed with RPMI-1640 medium and added to the 6-well plates. After $12 \mathrm{~h}$, the medium was replaced with fresh medium. After a further $48 \mathrm{~h}$, the cells were observed under a fluorescence microscope (Olympus CKX41SF; Olympus Corp., Tokyo, Japan), in order to determine the percentage of cells synthesizing green fluorescent protein (GFP). The ratio of the number of cells that emit green fluorescence to the same field of view is the transfection efficiency. We then detected the protein expression of SIRT1 in the transfected mouse AECs-II by western blot analysis.

Exposure of cells to $P Q$. The AECs-II were either exposed to various concentrations of PQ $(0,200,400,800$ and $1000 \mu \mathrm{M})$ for $24 \mathrm{~h}$ or to $800 \mu \mathrm{M}$ PQ for various periods of time $(6,12,24$ and $48 \mathrm{~h}$ ). The control cells were untreated cells.

Western blot analysis. After harvesting, the mouse AECs-II were lysed on ice using lysis buffer (Beyotime Biotechnology, Jiangsu, China) for $30 \mathrm{~min}$. Subsequently, the cell lysates were centrifuged at $16,000 \mathrm{x} \mathrm{g}$ for $15 \mathrm{~min}$ at $4^{\circ} \mathrm{C}$. The supernatants were collected and the protein concentration was detected using the bicinchoninic acid (BCA) method. Equal amounts of protein (40 $\mu \mathrm{g} /$ lane) were loaded onto a polyacrymide gel and run at $80 \mathrm{~V}$ for $1 \mathrm{~h}$, followed by a $120 \mathrm{~V}$ run for $30 \mathrm{~min}$, and then transferred to polyvinylidene fluoride (PVDF) membranes (0.45 $\mu \mathrm{m}$; Millipore, Billerica, MA, USA) by electroblotting. After blocking with 5\% skimmed powdered milk for $2 \mathrm{~h}$, the membranes were correspondingly incubated with antiSIRT1 $(1: 1,000)$, anti-NRF2 (1:1,000) or anti-GAPDH $(1: 5,000)$ antibodies overnight at $4^{\circ} \mathrm{C}$. The membranes were then washed in Tris-buffered saline-Tween-20 (TBST) 3 times for 10 min each and further incubated with horseradish peroxidase (HRP)-conjugated goat anti-rabbit secondary antibodies (Biogot Technology, Co., Ltd., Shanghai, China) for $2 \mathrm{~h}$ at room temperature and detected using an ECL detection system (Santa Cruz Biotechnology, Inc., Santa Cruz, CA, USA).

Determination of SOD and catalase (CAT) activities, as well as glutathione (GSH) and malondialdehyde (MDA) levels. Following exposure to $\mathrm{PQ}$, either supernatant or cell samples were collected. The SOD and CAT activities were detected according to the manfacturer's instructions (provided with the SOD and CAT assay kits; Jiancheng Bioengineering Institute, Nanjing, China). We also measured the MDA and GSH levels following the instructions provided by the manufacturer (Jiancheng Bioengineering Institute). The samples were analyzed using a spectrophotometer. The SOD and CAT activities were expressed in U/mgprot. The GSH levels were expressed in $\mu \mathrm{mol} / g$ prot and the MDA levels were expressed in $\mathrm{nmol} / \mathrm{mgprot}$.

Determination of HO-1 activity by ELISA. The cell supernatants were harvested following exposure of the cells to PQ. HO-1 activity was measured using commercially available enzyme-linked immunosorbent assay (ELISA) kits (Westang Biotechnology, Shanghai, China). ELISA was performed 


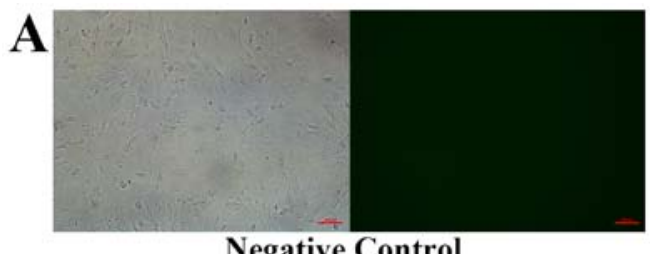

Negative Control
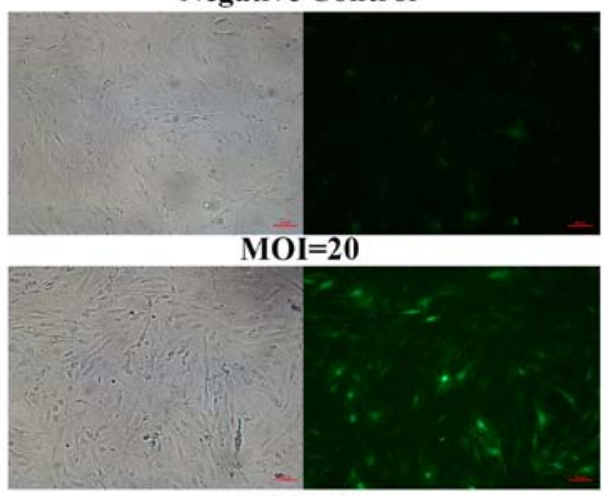

MOI $=80$
B
SIRT1
GAPDH
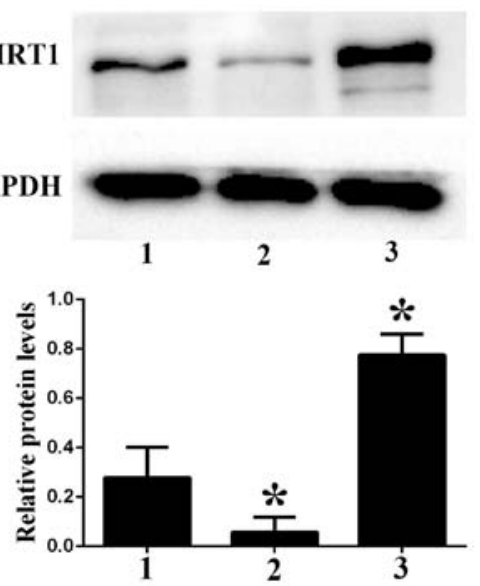

Figure 1. Transfection efficiency analysis. Mouse AECs-II were transfected with a lentiviral overexpression vector (LV-SIRT1) or lentiviral vector carrying shRNA targeting SIRT1 (shRNA-SIRT1). (A) After $72 \mathrm{~h}$, the cells were observed under a fluorescence microscope. (B) The protein expression of SIRT1 was measured by western blot analysis. The values were expressed as the means \pm SD ( $n=4$ experiments). Bar 1, control group; bar 2 , shRNA-SIRT1 group; bar 3 , LV-SIRT1 group. * $\mathrm{p}<0.05$, statistically significant difference compared to the control group.

strictly according to the manufacturer's instructions. The samples were analyzed using a spectrophotometer. HO-1 activity was expressed in $\mu \mathrm{g} / \mathrm{gprot}$.

Analysis of cell apoptosis. The cell apoptotic rate was detected using a FACScan flow cytometer (Becton-Dickinson, Franklin Lakes, NJ, USA) with an Annexin V-FITC/propidium iodide (PI) apoptosis detection kit (Nanjing Keygen Biotechnology Co. Ltd., Nanjing, China). Following exposure to PQ, the cells were harvested, washed with ice-cold PBS and then re-suspended in $500 \mu \mathrm{l}$ binding buffer at a concentration of $1 \times 10^{6}$ cells $/ \mathrm{ml}$. Subsequently, the cells were stained with $5 \mu 1$ of Annexin V-FITC and $5 \mu \mathrm{l}$ of PI, followed by incubation for $15 \mathrm{~min}$ at room temperature in the dark. Finally, apoptosis was assessed by flow cytometry.

Analysis of protein stability. The cells were seeded in 6-well plates and exposed to $800 \mu \mathrm{M}$ PQ for $24 \mathrm{~h}$. Subsequently, the culture solution was changed to cycloheximide solution (CHX Sigma-Aldrich) at a concentration of $100 \mathrm{~g} / \mathrm{ml}$ and treated for $0,6,12$ and $24 \mathrm{~h}$. The treated cells were collected and processed for western blot analysis following the removal of cycloheximide.

Detection of acetylated NRF2 expression in cells. After harvesting, the whole cell lysates were prepared using non-denaturing lysis buffer (Thermo Fisher Scientific) on ice and immunoprecipitation was performed with the whole cell lysates. Briefly, the lysates were incubated with $1 \mu \mathrm{g}$ each of either normal rabbit IgG (Cell Signaling Technology) or anti-NRF2 at $4^{\circ} \mathrm{C}$ with gentle rotation overnight and collected by incubating with Protein A/G Plus Agarose (Thermo Fisher Scientific). The agarose beads were washed and then boiled for 10 min with 1 X SDS loading buffer followed by centrifuga- tion at $1,000 \mathrm{x} g$ for $1 \mathrm{~min}$. The immunocomplexes were then detected by western blot analysis using anti-acetyl-lysine antibody (\#9441; Cell Signaling Technology).

Statistical analysis. All data are presented as the means \pm SD and were analyzed using SPSS 19.0 software (SPSS Inc., Chicago, IL, USA). GraphPad Prism 6 (GraphPad Software Inc., La Jolla, CA, USA) was used to prepare the figures. The comparisons between groups were analyzed using one-way ANOVA. A p-value $<0.05$ was considered to indicate a statistically significant difference.

\section{Results}

Transfection efficiency analysis. As shown in Fig. 1A, the cells in the negative control group cells did not express green fluorescence. Under the condition of a multiplicity of infection (MOI) of 20, the transfection efficiency of the lentivirus in the mouse AECs-II is very low. When the MOI was increased to 80 , the transfection efficiency of the lentivirus in the mouse AECs-II increased by $>80 \%$. Thus, in all the experiments, the lentivirus was transfected at an MOI of 80. As shown in Fig. 1B, compared with the control group, SIRT1 protein expression in the shRNA-SIRT1 group decreased significantly, while that in the LV-SIRT1 group significantly increased (all $\mathrm{p}<0.05$ ).

$P Q$ induces the apoptosis of mouse AECs-II. The proportion of early apoptotic and late apoptotic cells was counted. As shown in Fig. 2, following exposure to increasing concentrations of $\mathrm{PQ}$, the proportion of early apoptotic cells increased; however, when the concentration reached $1000 \mu \mathrm{M}$, the percentage of late apoptotic cells increased significantly more than the proportion of early apoptotic cells at the same time. Similarly, in the groups of cells exposed to a PQ concentration of $800 \mu \mathrm{M}$ 

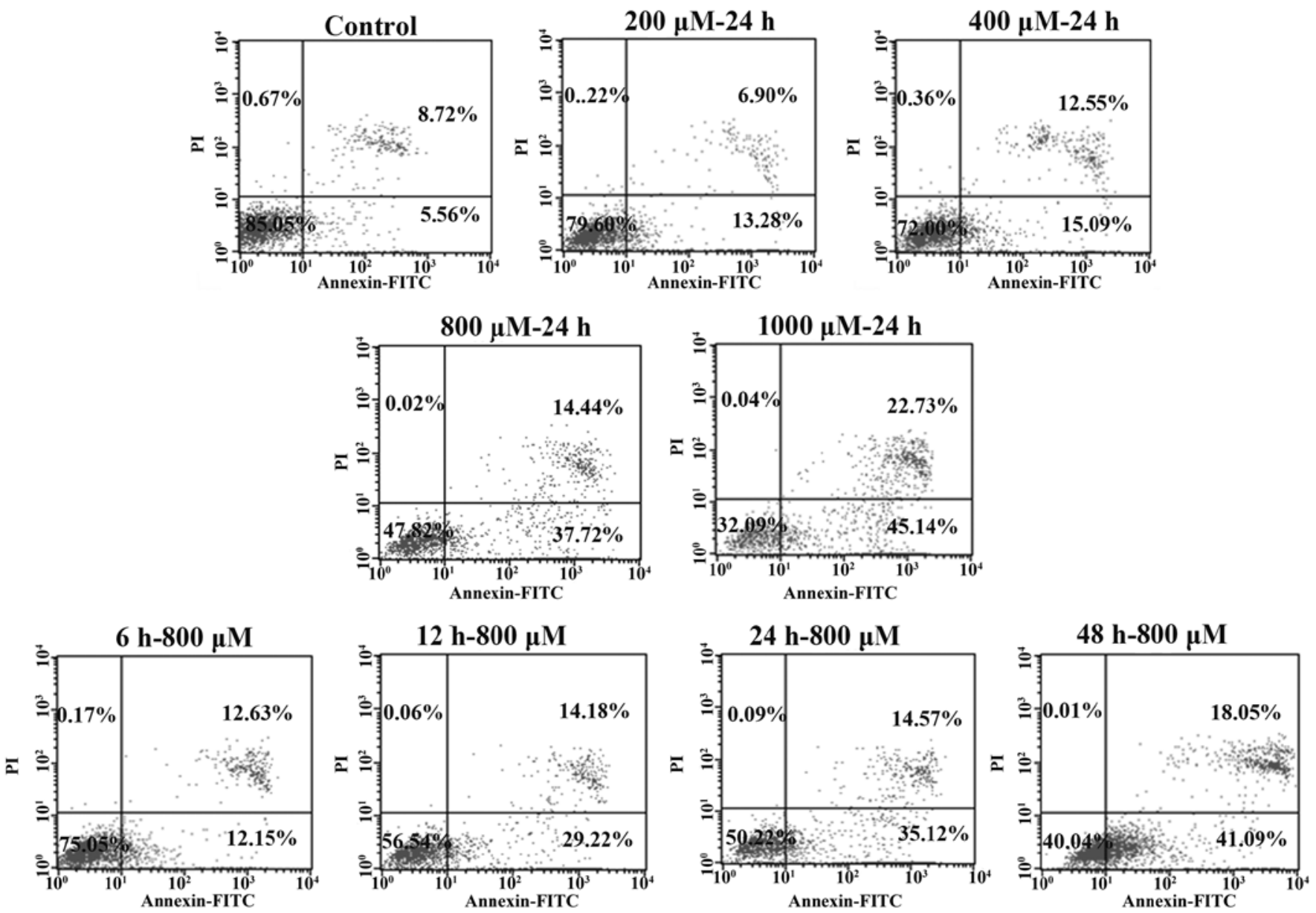

Figure 2. Paraquat (PQ)-induced apoptosis of mouse type II alveolar epithelial cells (AECs-II). The mouse AECs-II were treated with various concentrations of PQ $(0,200,400,800$ and $1000 \mu \mathrm{M}$; top and middle panels) for $24 \mathrm{~h}$, and also treated with $800 \mu \mathrm{M}$ PQ for 6, 12, 24 and $48 \mathrm{~h}$ (bottom panels). The apoptosis of the cells was detected using a FACSCalibur flow cytometer. The 4 quadrants indicate the following: left upper quadrant, mechanically broken cells; left lower quadrant, surviving cells; upper right quadrant, late apoptotic cells; lower right quadrant, early apoptotic cells.

for different periods of time, the proportion of apoptotic cells also increased in a time-dependent manner.

Protein expression of SIRT1 and NRF2 in mouse AECs-II following exposure to $P Q$. To assess the potential roles of SIRT1 and NRF2 in PQ-induced lung injury, the protein levels of SIRT1 and NRF2 were measured by western blot analysis in the mouse AECs-II following exposure to various concentrations of PQ for $24 \mathrm{~h}$ (Fig. 3A) and exposure to $800 \mu \mathrm{M}$ PQ for different periods of time (Fig. 3B). The results revealed that exposure of the cells to low concentations (200 and $400 \mu \mathrm{M}$ ) of PQ upregulated the protein expression of SIRT1 and NRF2. With the increasing concentations $(800$ and $1000 \mu \mathrm{M})$ of PQ, the protein expression of SIRT1 and NRF2 gradually decreased. Furthermore, during the short periods of exposure to $\mathrm{PQ}$, the protein expression of SIRT1 and NRF2 was significantly elevated and peaked at $12 \mathrm{~h}$. As the duration of exposure to PQ was extended, the protein expression of SIRT1 and NRF2 gradually decreased.

Effects of $P Q$ on SOD, CAT and HO-1 activities, as well as on GSH and MDA levels in mouse AECs-II. Following exposure to low concentrations of PQ $(200$ and $400 \mu \mathrm{M})$ for $24 \mathrm{~h}$ (Fig. 4A-D), the SOD, CAT and HO-1 activities, as well as the GSH levels significantly increased compared with the control groups. As the PQ concentration increased (800 and $1000 \mu \mathrm{M}$ ), the activities of SOD, CAT and HO-1, as well as the levels of GSH gradually decreased. The levels of MDA (Fig4E) in the cells increased in a PQ concentration-dependent manner.

Following a 12-h challenge with $800 \mu \mathrm{M}$ PQ (Fig. 4F-H), the GSH levels and CAT activity significantly increased compared with the untreated control cells, reaching peak levels. SOD activity reached a peak at $6 \mathrm{~h}$. With the extension of the duration of exposure to PQ, the activities of SOD and CAT, as well as the GSH levels were markedly decreased compared with the control groups. The levels of MDA and HO-1 (Fig. 4I and J) in the cells increased in a time-dependent manner.

Overexpression of SIRT1 inhibits the apoptosis of AECs-II. Following the knockdown or upregulation of SIRT1 expression, the mouse AECs-II were challenged with $800 \mu \mathrm{M}$ PQ for $24 \mathrm{~h}$. The overexpression of SIRT1 significantly decreased the cell apoptotic rate. On the contrary, the downregulation of SIRT1 enhanced the apoptotic rate of the cells (Fig. 5A-D).

SIRT1 protein suppresses $P Q$-induced oxidative stress in mouse AECs-II. Compared with normal conditions, SOD 
A

SIRT1

NRF2

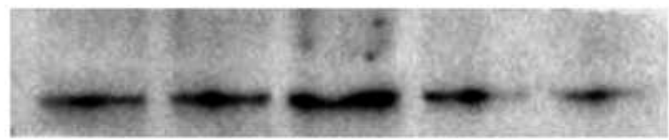

GAPDH

Control $200 \mu \mathrm{M} \quad 400 \mu \mathrm{M} \quad 800 \mu \mathrm{M} 1000 \mu \mathrm{M}$

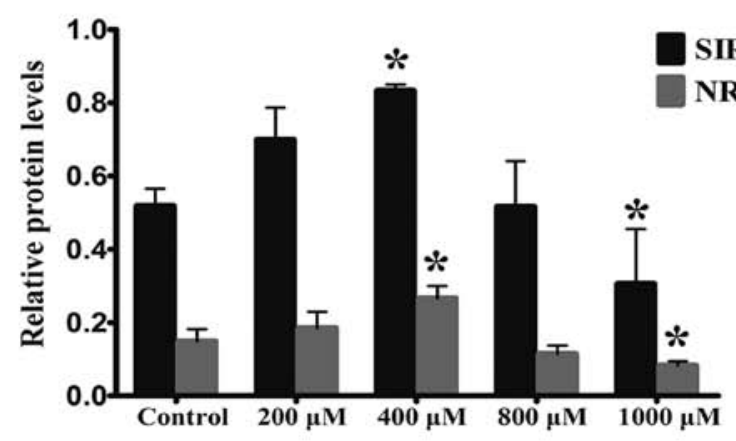

B

\section{SIRT1}

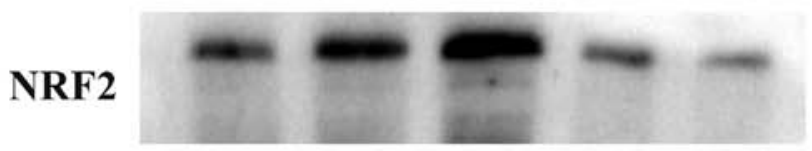

\section{GAPDH}
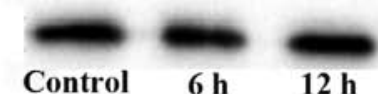

$24 \mathrm{~h}$

$48 \mathrm{~h}$

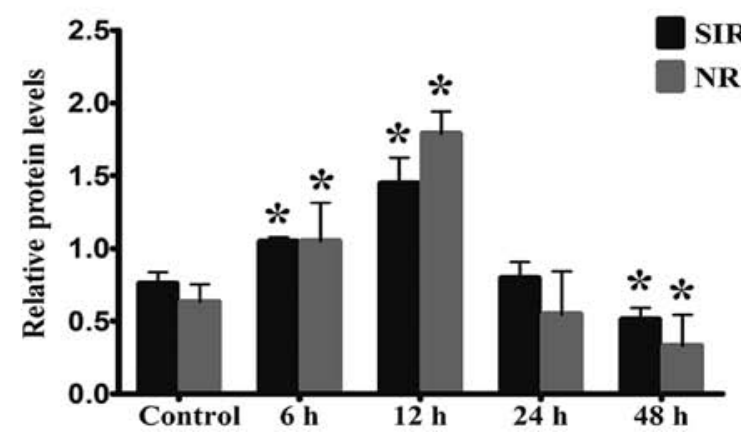

Figure 3. Protein expression of silent information regulator 2-related enzyme 1 (SIRT1), nuclear factor E2-related factor 2 (NRF2) in mouse type II alveolar epithelial cells (AECs-II) following exposure to paraquat (PQ). Mouse AECs-II were treated with (A) 200, 400, 800 and $1000 \mu \mathrm{M}$ PQ for $24 \mathrm{~h}$ and (B) also treated with $800 \mu \mathrm{M}$ PQ for $6,12,24$ and $48 \mathrm{~h}$. The protein expression of SIRT1 and NRF2 was measured by western blot analysis. The values are expressed as the means $\pm \mathrm{SD}\left(\mathrm{n}=4\right.$ experiments). ${ }^{*} \mathrm{p}<0.05$, statistically significant difference when compared with the control group.

and CAT activity, as well as the GSH levels were markedly inhibited in the mouse AECs-II following exposure to $800 \mu \mathrm{M}$ PQ (Fig. 5E-G). The silencing of SIRT1 by shRNA targeting SIRT1 (shRNA-SIRT1) suppressed the activity of SOD and
CAT, as well as the GSH levels to an even greater extent compared with the cells epxosed to PQ and not transfected with shRNA, whereas the overexpression of SIRT1 using a SIRT1 overexpression vector (LV-SIRT1) markedly increased the activity/levels of these enzymes (Fig. 5E-G). Conversely, the MDA levels and HO-1 activity (Fig. 5H and I) were notably elevated in the mouse AECs-II following exposure to PQ. The silencing of SIRT1 increased the expression of MDA, whereas the upregulation of SIRT1 had the opssosite effect. However, the overexpression of SIRT1 promoted HO-1 activation and upregulated its expression.

SIRT1 protein expression upregulates NRF 2 protein expression. The mouse AECs-II in which SIRT1 was either overexpressed or knocked down were exposed to PQ and harvested at $24 \mathrm{~h}$. As shown in Fig. 6A and B, the overexpression of SIRT1 promoted the expression of NRF2, whereas the knockdown of SIRT1 blocked NRF2 activation. NRF2 expression correlated with the levels of SIRT1.

SIRT1 protein enhances the stability of NRF2 protein. The stability of NRF2 protein expression gradually decreased following exposure of the cells to PQ for $6 \mathrm{~h}$. In the control group (Fig. 6E and I), the expression of NRF2 decreased by 24.7 and $42.51 \%$ at 12 and $24 \mathrm{~h}$, respectively. In the PQ group (Fig. 6F and I), NRF2 expression decreased by 53.77 and $71.6 \%$, following treatment with $\mathrm{CHX}$ for 12 and $24 \mathrm{~h}$, respectively. In the PQ + shRNA-SIRT1 group (Fig. 6G and I), the downregulation of SIRT1 significantly damaged the stability of NRF2, with the decrease in expression reaching 71.7 and $74.8 \%$ at 12 and $24 \mathrm{~h}$, respectively. In the PQ + LV-SIRT1 group (Fig. $6 \mathrm{H}$ and I), the upregulation of SIRT1 protein expression played a negative role in the degradation of NRF2 protein, with the decline only reaching 41.0 and $47.9 \%$ at the corresponding time points.

SIRT1 protein deacetylates NRF2 protein in mouse AECs-II. To determine whether SIRT1 plays a vital role in the acetylation of NRF2 protein, the levels of acetylated NRF2 in the cells were detected. Our data (Fig. 6C and D) revealed that the NRF2 protein acetylation level was markedly upregulated by the depletion of SIRT1, whereas the overexpression of SIRT1 reduced the NRF2 acetylation level.

\section{Discussion}

Previous studies have reported that PQ-induced pulmonary toxicity directly results in bronchial toxicity, inflammation and oxidative stress $(18,19,20)$. It has been reported that excessive amounts of ROS-mediated oxidative stress play an important role in the process of PQ-induced pulmonary injury $(21,22)$. $\mathrm{PQ}$ is an influential inducer of ROS and may prompt the generation of ROS, such as superoxide anions, as well as hydroxyl and peroxyl radicals $(1,23)$. After cellular uptake, PQ has been found to target the mitochondria and through cyclic reduction-oxidation reactions, causes an increase in intracellular ROS generation, which then leads to the apoptosis of cells $(10,24)$. The findings of the present study revealed that exposure of the cells to high concentrations of $\mathrm{PQ}$ induced a significant increase in the MDA content, whereas the activi- 

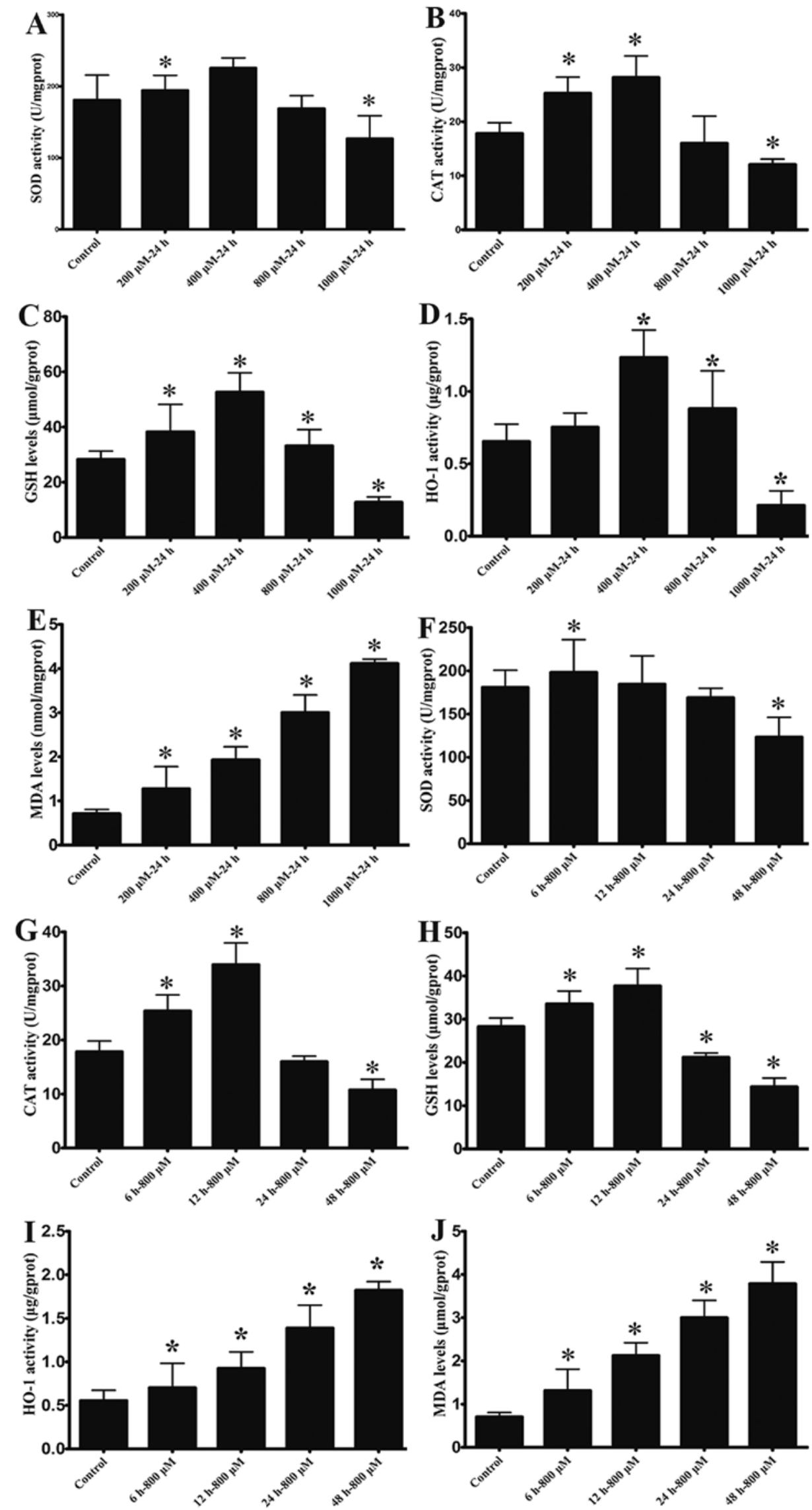

Figure 4. Effect of paraquat (PQ) on superoxide dismutase (SOD), catalase (CAT) and heme oxygenase-1 (HO-1) activities, as well as on glutathione (GSH) and malondialdehyde (MDA) levels in mouse type II alveolar epithelial cells (AECs-II). The mouse AECs-II were treated with (A-E) $200,400,800$ and $1000 \mu \mathrm{M}$ PQ for $24 \mathrm{~h}$ and (F-J) also treated with $800 \mu \mathrm{M}$ PQ for 6, 12, 24 and $48 \mathrm{~h}$. The activities of SOD and CAT, and the levels of GSH, MDA and HO-1 were respectively measured using the corresponding detection kits. The values are expressed as the means $\pm \mathrm{SD}$ ( $\mathrm{n}=4$ experiments). ${ }^{*} \mathrm{p}<0.05$, statistically significant difference when compared with the control group. 

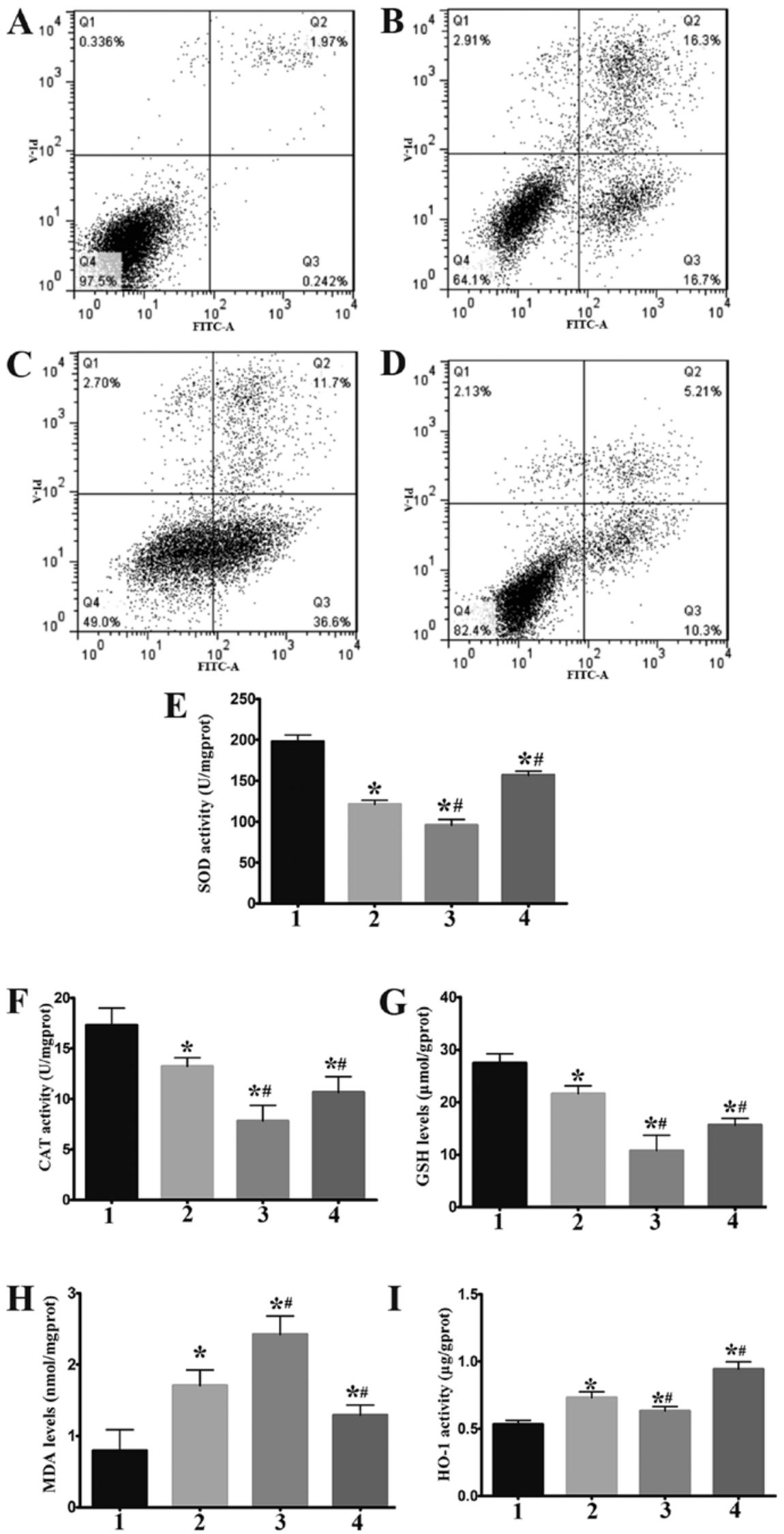

Figure 5. Silent information regulator 2-related enzyme 1 (SIRT1) protein inhibits paraquat (PQ)-induced apoptosis and suppresses oxidative stress in mouse type II alveolar epithelial cells (AECs-II). Transfected mouse AECs-II were exposed to $800 \mu \mathrm{M}$ PQ for $24 \mathrm{~h}$. (A-D) The apoptosis of the cells was detected using a FACSCalibur flow cytometer. (A) Control group; (B) PQ group; (C) PQ + shRNA-SIRT1 group; (D) PQ + LV-SIRT1 group. (E-I) The activities of superoxide dismutase (SOD), catalase (CAT), heme oxygenase-1 (HO-1), and the levels of glutathione (GSH) and malondialdehyde (MDA) were detected using the corresponding detection kits. The values are presented as the means $\pm \mathrm{SD}$ ( $\mathrm{n}=4$ experiments). Bar 1, control group; bar 2, PQ group; bar 3, PQ + shRNA-SIRT1 group; bar 4, PQ + LV-SIRT1 group. " $\mathrm{p}<0.05$, statistically significant difference when compared with the control group; ${ }^{*} \mathrm{p}<0.05$, statistically significant difference when compared with the PQ group. 


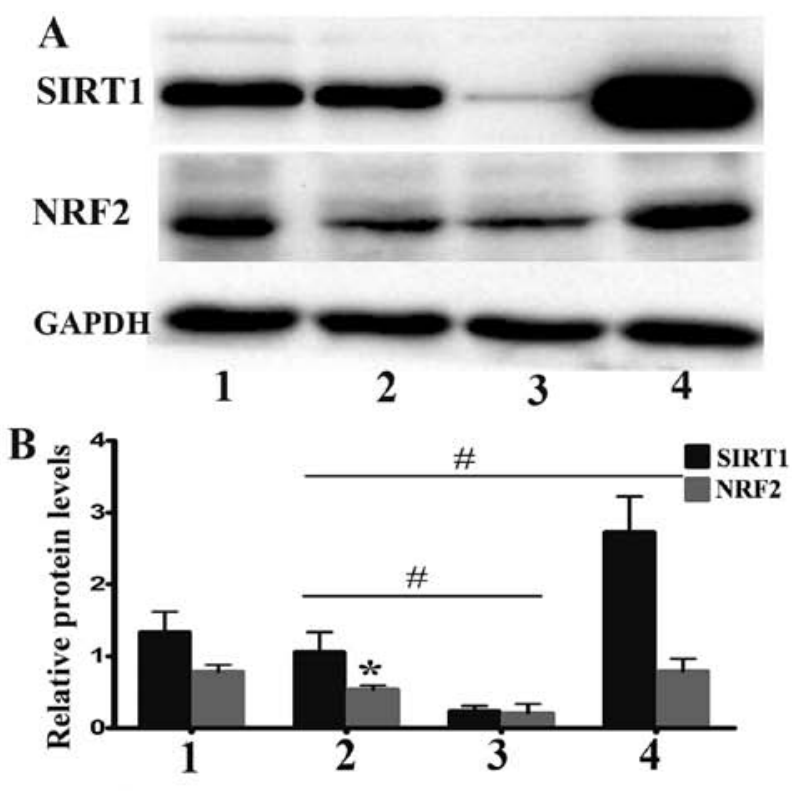

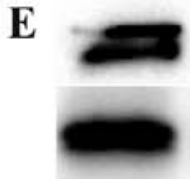

$\mathbf{0 ~ h}$

F

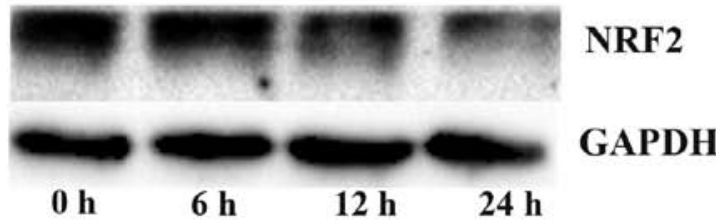

G

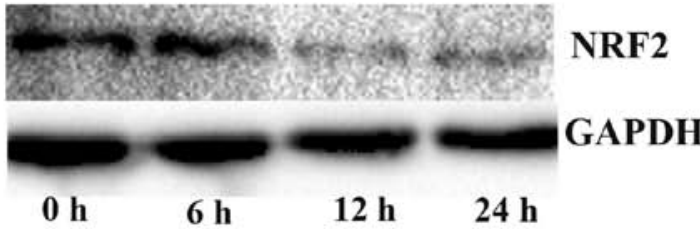

$\mathbf{H}$
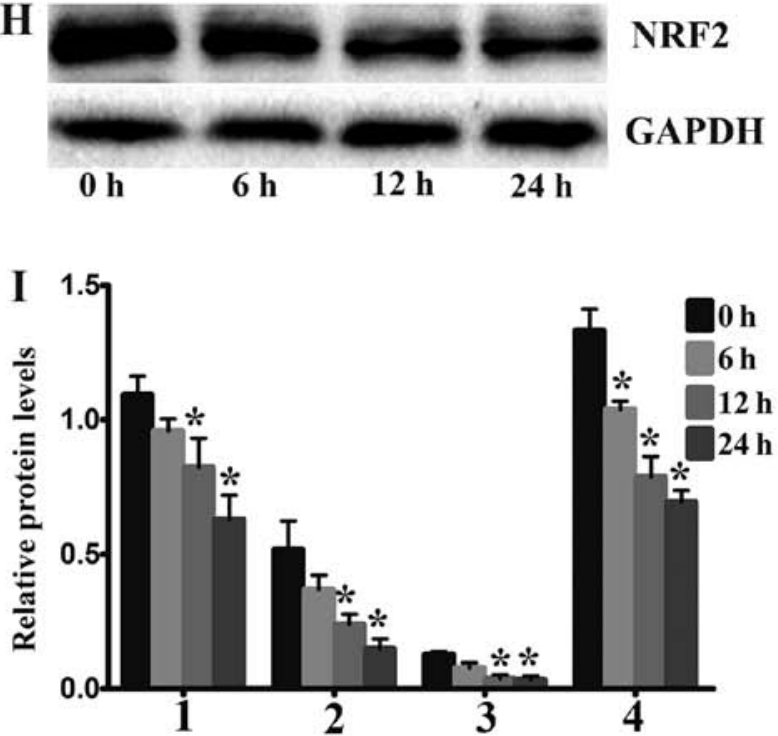

Figure 6. Silent information regulator 2-related enzyme 1 (SIRT1) protein upregulates the expression and stability of nuclear factor E2-related factor 2 (NRF2) protein by deacetylating NRF2. Mouse type II alveolar epithelial cells (AECs-II) were treated with $800 \mu \mathrm{M}$ paraquat (PQ) for $24 \mathrm{~h}$ after successful transfection. (A and B) The protein expression levels of SIRT1 and NRF2 were then measured by western blot analysis. (E-I) Transfected mouse AECs-II were treated with $100 \mu \mathrm{g} / \mathrm{ml}$ of cycloheximide (CHX) for $0,6,12$ and $24 \mathrm{~h}$ following exposure to PQ. The protein stability of NRF2 was measured by western blot analysis. (E) Control group; (F) PQ group; (G) PQ + shRNA-SIRT1 group; (H) PQ + LV-SIRT1 group. (C and D) Following successful transfection, the NRF2 protein was immunoprecipitated with anti-NRF2 antibody. The acetylated NRF2 protein was detected by western blot analysis using anti-acetyl-lysine antibody. Lane 1, Control group; lane 5, shRNA-SIRT1, group; lane 6, LV-SIRT1 group. The values are presented as the means $\pm \mathrm{SD}$ ( $\mathrm{n}=4$ experiments). " $\mathrm{p}<0.05$, statistically significant difference when compared with the control group; $\mathrm{p}<0.05$, statistically significant difference when compared with the PQ group. Lane or bar 1, control group; lane or bar 2 , PQ group; lane or bar 3, PQ + shRNA-SIRT1 group; lane or bar 4, PQ + LV-SIRT1 group; lane or bar 5, shRNA-SIRT1 group; lane or bar 6, LV-SIRT1 group.

ties of SOD and CAT were decreased, which was consistent with the findings of a previous study (25).

In a previous study, NRF2 was considered as an effective target for the antioxidant therapy of PQ poisoning (15). The levels of oxidative stress were assessed by measuring the levels/activity of MDA, SOD, GSH and CAT, which were also associated with its anti-apoptotic properties (26). It has been proven that NRF2 expression maintains the balance of the oxidant-antioxidant system and inhibits inflammation, as well as apoptotic factors, which are associated with an anti-apoptotic effect on cell injury induced by PQ (27). As NRF2 may activate cellular responses through enhanced transcription by antioxidant response element (ARE), this leads to increases in the expression levels of antioxidant enzymes. including CAT, 
SOD, GSH and glutathione peroxidase (GPx) $(15,28)$. It has been demonstrated that the ability to eliminate electrophiles and free radicals is enhanced by these defensive proteins, which collectively promotes the detoxification of ROS, and increases the capacity of cells to resist apoptosis $(12,29)$. Previous studies have confirmed that the depletion of NRF2 by siRNA downregulates the expression of SOD and upregulates the levels of MDA and apoptotic factors, including phosphorylated p53, Bax and the active form of caspase-3, which significantly enhances PQ-induced oxidative damage in cells in vitro $(18,30)$. Moreover, NRF2 enhances the inhibitory effects of HO-1 on the intracellular production of ROS (31). Additionally, it has been demonstrated that the overexpression of NRF2 effectively alleviates PQ-induced lung injury and cell apoptosis (32). In the present study, we verified that NRF2 overexpression induced by transfection with LV-SIRT1 played an important protective role by reducing the cell apoptosis induced by PQ. This study provides evidence of the involvement of the NRF2 signaling pathway in the antioxidant effects exerted by SIRT1, thereby improving the endogenous cellular anti-apoptotic system.

In unstressed cells, NRF2 has been found to be sequestered by its inhibitor, Keap1, thereby promoting rapid proteasome-mediated degradation (33). However, in response to oxidative stress, NRF2 dissociates from Keap1, through modifications to the Keap1-NRF2 complex, such as ubiquitination, proteasomal degradation or phosphorylation (34). NRF2 has been found to be regulated by acetylation; the results of a previous study indicated that NRF2 acetylation at lysine residues in the Neh1 DNA-binding domain was responsible for NRF2 transactivation rather than for stability (35). However, a previous study also demonstrated that the downregulation of histone deacetylase (HDAC)2 may cause the impaired functioning of NRF2, which leads to the reduced expression of the antioxidant responsive genes and thus, it was concluded that HDAC2 may regulate NRF2 activity through deacetylation (36). In this study, we found that SIRT1 deacetylated NRF2 in cells, thus increasing its stability, resulting in the enhanced expression of antioxidant genes and decreased sensitivity to oxidative stress.

It is well known that sirtuins are involved in various aspects of biological processes through deacetylation, such as the regulation of gene expression, cellular stress response and DNA repair (37). There is a close association between SIRT1 expression and oxidative stress. Cigarette smoke-mediated oxidative stress has been reported to decrease SIRT1 protein levels and activity in the lungs of rats and human beings $(4,38)$. In the present study, SIRT1 overexpression was associated with a high level of GSH, as well as SOD and CAT activities, the downstream genes of NRF2-anti-ARE, whereas the depletion of SIRT1 markedly increased the activity of MDA and HO-1, which suggested that SIRT1 protein may play an important role in mediating the oxidant-antioxidant balance in PQ-induced pulmonary injury (39) and enhanced cell resistance to oxidative stress. In a previous study of ours (40), resveratrol, an activator of SIRT1, increased the SIRT1 and NRF2 levels, and through the activation of the NRF2-ARE pathway led to the suppression of ROS production.. In that study, we demonstrated that PQ stimulated the compensatory overexpression of SIRT1 and NRF2, whereas exposure to PQ for a longer period of time significantly decreased the expression of SIRT1, as well as
NRF2 in the lungs of mice. Additionally, treatment with resveratrol not only upregulated the expression of SIRT1 and NRF2, but also increased the activities of HO-1, SOD and CAT. The depletion of SIRT1 has also been shown to block NRF2-ARE pathway activation and reverse the antioxidative capacity (15). In this study, our results revealed that the upregulation of SIRT1 expression markedly increased the expression of NRF2, whereas the knockdown of SIRT1 had a negative influence on the levels of NRF2 expression. Therefore, we hypothesized that SIRT1 protein would alleviate PQ-induced oxidative stress through the NRF2 pathway. It has previously been confirmed that SIRT1 promotes nuclear accumulation, DNA binding and the transcriptional activities of NRF2 and promotes the expression of the NRF2 downstream genes, HO-1, and SOD, resulting in the reduction of ROS levels in a deacetylase-dependent manner (14). Importantly, SIRT1 is a redox-sensitive deacetylase that is post-translationally modified by oxidants and carbonyl stress (4). We found that the upregulation of SIRT1 caused NRF2 deacetylation, resulting in the increased stability and activity of NRF2, thus causing the suppression of apoptosis and resistance to oxidative stress.

Collectively, our results demonstrated that SIRT1 enhanced and stabilized NRF2 protein expression. We demonstrated that SIRT1 was associated with NRF2 and prevents its degradation possibly through the deacetylation of various residues. As the reduced SIRT1 activity is observed in PQ poisoning due to oxidative stress, this may consequently reduce the activity of NRF2, thus inhibiting an increase in the expression of antioxidant enzymes; this in turn increases oxidative stress, which then further reduces SIRT1 activity; this process is an ongoing vicious circle. SIRT1 overexpression in cells may upregulate Nrf2 expression by increasing Nrf2 deacetylation, resulting in the enhancement of normal antioxidant defences.

\section{Acknowledgements}

This study was supported by the State Key Program of Natural Science Foundation of Zhejiang province (no. LZ12H26001), and the Medical and Health Research Program of Zhejiang province (no. 2012ZDA034).

\section{References}

1. Gawarammana IB and Buckley NA: Medical management of paraquat ingestion. Br J Clin Pharmacol 72: 745-757, 2011.

2. Shao X and Chen JH: Progress on pathogenesis and treatment of paraquat-induced pulmonary fibrosis. Zhejiang Da Xue Xue Bao Yi Xue Ban 43: 717-727, 2014 (In Chinese).

3. He X, Wang L, Szklarz G, Bi Y and Ma Q: Resveratrol inhibits paraquat-induced oxidative stress and fibrogenic response by activating the nuclear factor erythroid 2-related factor 2 pathway. J Pharmacol Exp Ther 342: 81-90, 2012.

4. Caito S, Rajendrasozhan S, Cook S, Chung S, Yao H, Friedman AE, Brookes PS and Rahman I: SIRT1 is a redoxsensitive deacetylase that is post-translationally modified by oxidants and carbonyl stress. FASEB J 24: 3145-3159, 2010.

5. Salminen A, Kaarniranta K and Kauppinen A: Crosstalk between oxidative stress and SIRT1: impact on the aging process. Int J Mol Sci 14: 3834-3859, 2013.

6. Yao H, Sundar IK, Huang Y, Gerloff J, Sellix MT, Sime PJ and Rahman I: Disruption of sirtuin 1-mediated control of circadian molecular clock and inflammation in chronic obstructive pulmonary disease. Am J Respir Cell Mol Biol 53: 782-792, 2015.

7. Chun P: Role of sirtuins in chronic obstructive pulmonary disease. Arch Pharm Res 38: 1-10, 2015. 
8. Yao H, Hwang JW, Sundar IK, Friedman AE, McBurney MW, Guarente L, Gu W, Kinnula VL and Rahman I: SIRT1 redresses the imbalance of tissue inhibitor of matrix metalloproteinase-1 and matrix metalloproteinase-9 in the development of mouse emphysema and human COPD. Am J Physiol Lung Cell Mol Physiol 305: L615-L624, 2013.

9. Hong GL, Cai QQ, Tan JP, Jiang XZ, Zhao GJ, Wu B, Li MF, Qiu QM and Lu ZQ: Mifepristone-inducible recombinant adenovirus attenuates paraquat-induced lung injury in rats. Hum Exp Toxicol 34: 32-43, 2015.

10. Hong GL, Liu JM, Zhao GJ, Wang L, Liang G, Wu B, Li MF, Qiu QM and Lu ZQ: The reversal of paraquat-induced mitochondria-mediated apoptosis by cycloartenyl ferulate, the important role of Nrf2 pathway. Exp Cell Res 319: 2845-2855, 2013.

11. Dinkova-Kostova AT: The role of sulfhydryl reactivity of small molecules for the activation of the KEAP1/NRF2 pathway and the heat shock response. Scientifica (Cairo) 2012: 606104, 2012.

12. Giudice A, Arra C and Turco MC: Review of molecular mechanisms involved in the activation of the Nrf2-ARE signaling pathway by chemopreventive agents. Methods Mol Biol 647: 37-74, 2010.

13. Motohashi $\mathrm{H}$ and Yamamoto M: Nrf2-Keap1 defines a physiologically important stress response mechanism. Trends Mol Med 10: 549-557, 2004

14. Huang K, Huang J, Xie X, Wang S, Chen C, Shen X, Liu P and Huang H: Sirtl resists advanced glycation end products-induced expressions of fibronectin and TGF- $\beta 1$ by activating the Nrf2/ARE pathway in glomerular mesangial cells. Free Radic Biol Med 65: 528-540, 2013.

15. Huang K, Chen C, Hao J, Huang J, Wang S, Liu P and Huang H: Polydatin promotes Nrf2-ARE anti-oxidative pathway through activating Sirt1 to resist AGEs-induced upregulation of fibronetin and transforming growth factor- $\beta 1$ in rat glomerular messangial cells. Mol Cell Endocrinol 399: 178-189, 2015.

16. Potteti HR, Rajasekaran S, Rajamohan SB, Tamatam CR, Machireddy N and Reddy SP: Sirtuin 1 promotes hyperoxiainduced lung epithelial death independent of NRF2 activation. Am J Respir Cell Mol Biol: Oct 14, 2015 (Epub ahead of print).

17. Cai Q, Lu Z, Hong G, Jiang X, Wu Z, Zheng J, Song Q and Chang Z: Recombinant adenovirus Ad-RUNrf2 reduces paraquat-induced A549 injury. Hum Exp Toxicol 31: 1102-1112, 2012.

18. Lu H, Chang Z, Han W, Wang L and Hong G: Curcumin reduces paraquat-induced oxidative injury in A549 cells by activation of the Nrf2-ARE pathway. Zhonghua Lao Dong Wei Sheng Zhi Ye Bing Za Zhi 32: 44-49, 2014

19. Dinis-Oliveira RJ, Pontes H, Bastos ML, Remião F, Duarte JA and Carvalho F: An effective antidote for paraquat poisonings: the treatment with lysine acetylsalicylate. Toxicology 255: 187-193, 2009.

20. Kim YS, Zerin T and Song HY: Antioxidant action of ellagic acid ameliorates paraquat-induced A549 cytotoxicity. Biol Pharm Bull 36: 609-615, 2013.

21. Yang SR, Park JR and Kang KS: Reactive oxygen species in mesenchymal stem cell aging: implication to lung diseases. Oxid Med Cell Longev 2015: p486263, 2015.

22. Toygar M, Aydin I, Agilli M, Aydin FN, Oztosun M, Gul H, Macit E, Karslioglu Y, Topal T, Uysal B and Honca M: The relation between oxidative stress, inflammation, and neopterin in the paraquat-induced lung toxicity. Hum Exp Toxicol 34: 198-204, 2015.

23. Wang $\mathrm{X}$, Luo $\mathrm{F}$ and Zhao $\mathrm{H}$ : Paraquat-induced reactive oxygen species inhibit neutrophil apoptosis via a p38 MAPK/NF- $\kappa$ B-IL-6/TNF- $\alpha$ positive-feedback circuit. PLoS One 9: e93837, 2014.

24. Dinis-Oliveira RJ, Duarte JA, Sánchez-Navarro A, Remião F, Bastos ML and Carvalho F: Paraquat poisonings: mechanisms of lung toxicity, clinical features, and treatment. Crit Rev Toxicol 38: 13-71, 2008.
25. Senator A, Rachidi W, Lehmann S, Favier A and Benboubetra M: Prion protein protects against DNA damage induced by paraquat in cultured cells. Free Radic Biol Med 37: 1224-1230, 2004.

26. Zhang S, Zhang Y, Li H, Xu W, Chu K, Chen L and Chen X: Antioxidant and anti-excitotoxicity effect of Gualou Guizhi decoction on cerebral ischemia/reperfusion injury in rats. Exp Ther Med 9: 2121-2126, 2015.

27. Jiang XZ, Song Q, Xu XP, Cai QQ, Hong GL, Liang H and Lu ZQ: The effects of Nrf2 gene expression induced by RU486 at different doses on A549 cell damage induced by paraquat. Zhonghua Lao Dong Wei Sheng Zhi Ye Bing Za Zhi 30: 268-272, 2012 (In Chinese).

28. Lee HJ, Han J, Jang Y, Kim SJ, Park JH, Seo KS, Jeong S, Shin S, Lim K, Heo JY and Kweon GR: Docosahexaenoic acid prevents paraquat-induced reactive oxygen species production in dopaminergic neurons via enhancement of glutathione homeostasis. Biochem Biophys Res Commun 457: 95-100, 2015.

29. Giudice A and Montella M: Activation of the Nrf2-ARE signaling pathway: a promising strategy in cancer prevention. Bioessays 28: 169-181, 2006.

30. Varì R, Scazzocchio B, Santangelo C, Filesi C, Galvano F, D'Archivio M, Masella R and Giovannini C: Protocatechuic acid prevents oxLDL-induced apoptosis by activating JNK/Nrf2 survival signals in macrophages. Oxid Med Cell Longev, p351827, 2015.

31. Ci X, Lv H, Wang L, Wang X, Peng L, Qin FX and Cheng G: The antioxidative potential of farrerol occurs via the activation of Nrf2 mediated HO-1 signaling in RAW 264.7 cells. Chem Biol Interact 239: 192-199, 2015 .

32. He XY, Zhao GJ, Lu ZQ, Hong GL, He F, Liang H, Qiu QM and Li JR: Oxidative stress of acute paraquat poisoned rats and sodium dimercaptopropane sulfonate intervention. Zhonghua Lao Dong Wei Sheng Zhi Ye Bing Za Zhi 27: 476-479, 2009 (In Chinese).

33. Kwak MK, Wakabayashi N, Itoh K, Motohashi H, Yamamoto M and Kensler TW: Modulation of gene expression by cancer chemopreventive dithiolethiones through the Keap1-Nrf2 pathway. Identification of novel gene clusters for cell survival. J Biol Chem 278: 8135-8145, 2003.

34. Baird L, Llères D, Swift S and Dinkova-Kostova AT: Regulatory flexibility in the Nrf2-mediated stress response is conferred by conformational cycling of the Keap1-Nrf2 protein complex. Proc Natl Acad Sci USA 110: 15259-15264, 2013.

35. Sun Z, Chin YE and Zhang DD: Acetylation of Nrf2 by p300/CBP augments promoter-specific DNA binding of Nrf2 during the antioxidant response. Mol Cell Biol 29: 2658-2672, 2009.

36. Mercado N, Thimmulappa R, Thomas CM, Fenwick PS, Chana KK, Donnelly LE, Biswal S, Ito K and Barnes PJ: Decreased histone deacetylase 2 impairs Nrf2 activation by oxidative stress. Biochem Biophys Res Commun 406: 292-298, 2011.

37. Yang WJ, Wang DL and Zhu WG: Mechanism of regulating deacetylase SIRT1 expression and activity. Yi Chuan 32: 1003-1008, 2010 (In Chinese).

38. Yao H, Sundar IK, Ahmad T, Lerner C, Gerloff J, Friedman AE, Phipps RP, Sime PJ, McBurney MW, Guarente L and Rahman I: SIRT1 protects against cigarette smoke-induced lung oxidative stress via a FOXO3-dependent mechanism. Am J Physiol Lung Cell Mol Physiol 306: L816-L828, 2014.

39. Tyagi N, Kumari A, Dash D and Singh R: Protective effects of intranasal curcumin on paraquot-induced acute lung injury (ALI) in mice. Environ Toxicol Pharmacol 38: 913-921, 2014.

40. Li S, Zhao G, Chen L, Ding Y, Lian J, Hong G and Lu Z: Resveratrol protects mice from paraquat-induced lung injury: The important role of SIRT1 and NRF2 antioxidant pathways. Mol Med Rep 13: 1833-1838, 2016. 